\title{
Estimating Target Heights Based on the Earth Curvature Model and Micromultipath Effect in Skywave OTH Radar
}

\author{
Hou Chengyu, Wang Yuxin, and Chen Jiawei \\ School of Electronics and Information Engineering, Harbin Institute of Technology, Harbin 150001, China \\ Correspondence should be addressed to Hou Chengyu; houcy@hit.edu.cn
}

Received 23 April 2014; Accepted 19 June 2014; Published 14 July 2014

Academic Editor: Weichao Sun

Copyright ( 2014 Hou Chengyu et al. This is an open access article distributed under the Creative Commons Attribution License, which permits unrestricted use, distribution, and reproduction in any medium, provided the original work is properly cited.

\begin{abstract}
Skywave over-the-horizon (OTH) radar systems have important long-range strategic warning values. They exploit skywave propagation reflection of high frequency signals from the ionosphere, which provides the ultra-long-range surveillance capabilities to detect and track maneuvering targets. Current OTH radar systems are capable of localizing targets in range and azimuth but are unable to achieve reliable instantaneous altitude estimation. Most existing height measurement methods of skywave OTH radar systems have taken advantage of the micromultipath effect and been considered in the flat earth model. However, the flat earth model is not proper since large error is inevitable, when the detection range is over one thousand kilometers. In order to avoid the error caused by the flat earth model, in this paper, an earth curvature model is introduced into OTH radar altimetry methods. The simulation results show that application of the earth curvature model can effectively reduce the estimation error.
\end{abstract}

\section{Introduction}

Skywave OTH radar works in the high frequency band (3$30 \mathrm{MHz}$ ), which uses the ionosphere to scatter the electromagnetic wave, observing the air and ground targets from top to bottom. Thus, skywave OTH radar has important longrange strategic warning value and a wide range of applications [1].

Skywave OTH radar systems are capable of localizing targets in range and azimuth but are unable to achieve reliable altitude estimation [2]. The altitude parameter is an important parameter for target recognition and tracking. Most existing altitude-finding theories for skywave OTH radar systems are based on the micromultipath effect. This effect refers to the fact that the radar signals have at least four kinds of paths through the relatively stable ionosphere and ground or sea surface reflection, when transmitted from the transmitting antenna and scattered by targets back to receiving antenna [3].

Currently, the methods that skywave OTH radar systems used to estimate the instantaneous altitude of the aircrafts can be divided into four kinds. (1) The superresolution method: this method uses modern spectral analysis method to distinguish echo delay of each propagation path and then estimates the target altitude. By this method, the height estimation is divided into three types: low altitude, intermediate altitude, and high altitude. But this technique can only be applied to analog data [3]. (2) The state-space multipath fading model: after a series of delays, the micromultipath echoes interfered by each other will cause distance-Doppler peak attenuation. Then, using of the Fourier analysis to the amplitude attenuation echoes, we can obtain altitude information [4]. (3) The high-resolution ionospheric sounder: through the experiment data by the high-resolution ionosphere sounder, the relation between the micromultipath effect and the target altitude is studied [5]. (4) The matched-field processing [6, 7]: the echo signal is used to rematch delay-Doppler space. And then the maximum likelihood estimation (MLE) is adopted to estimate the target altitude [8].

The methods mentioned above mostly consider the radar altimetry problem in the flat earth model. However, for skywave OTH radar systems, the detection range is thousands of kilometers, it will cause large errors to estimate target altitude with the flat earth model. Therefore, this paper will focus on how to introduce the earth curvature model into the altimetry method. 


\section{Signal Model and Maneuvering Target Model}

2.1. Establishing Echo Signal Model. In this paper, a monostatic multiple-input multiple-output (MIMO) radar system is considered, which consists of $N_{t}$ closely spaced transmit antennas and $N_{r}$ closely spaced receive antennas. Denote $S \in$ $C^{N t \times T}$ as the orthogonal narrowband waveform matrix which is transmitted from the $N_{t}$ transmit antennas over the pulse repetition period $T$. Assume that the orthogonal waveform is completed in the coherent integral time. $s_{i}$ is the $i$ th row of matrix $S$. When $i \neq m, s_{i}$ is orthogonal to $s_{m}$; then $S S^{H}=I_{N t}$. So, for the $k$ th pulse, the signal received at the $m$ th receive antenna is expressed as $[8,9]$

$$
u_{m, k}=\sum_{i=1}^{I} \sum_{n=1}^{N t} \rho_{i, k} s_{n} e^{j \phi_{i, m, k}^{[A]}} e^{j \phi_{i, n, k}^{[D]}}+w_{m, k},
$$

where $I=4$ is the number of the multipath components and $\rho_{i, k}$ is the complex response of the $i$ th multipath that considers the target reflection and propagation delays. The superscripts $[D]$ and $[A]$ denote the departure and the arrival modes, respectively. $d_{m}^{[A]}$ is the location of receive antennas. $\phi_{i, m, k}^{[A]}$ is determined by the $m$ th receive antenna and is expressed as

$$
\phi_{i, m, k}^{[A]}=\left(\frac{2 \pi}{\lambda}\right) d_{m}^{[A]} \sin \left(\theta_{i, k}^{[A]}\right),
$$

where $\theta_{i, k}^{[A]}$ is the elevation angle of arrival of the $i$ th path. Similarly, $\phi_{i, n, k}^{[D]}$ is determined by the location of the $n$th transmit antenna $d_{n}^{[D]}$, and $\phi_{i, n, k}^{[D]}$ is expressed as

$$
\phi_{i, n, k}^{[D]}=\left(\frac{2 \pi}{\lambda}\right) d_{n}^{[D]} \sin \left(\theta_{i, k}^{[D]}\right)
$$

where $\theta_{i, k}^{[D]}$ is the elevation angle of departure of the $i$ th path. For simplicity, we assume that the clutter has been filtered. Thus $w_{m, k}$ is the additive noise, which is independent and identically distributed complex Gaussian random variable and independent of the target echo signal.

So the $k$ th pulse echo, which is transmitted by the $m$ th transmit antenna and received by the $n$th receive antenna, can be expressed as

$$
r_{m, n, k}=\sum_{i=1}^{I} \rho_{i, k} e^{j \phi_{i, m, k}^{[A]}} e^{j \phi_{i, n, k}^{[D]}}+w_{m, n, k} .
$$

Denote $\varphi_{i, k}=\left[e^{\left(j \phi_{i, 1, k}^{[A]}+j \phi_{i, 1, k}^{[D]}\right)}, \ldots, e^{\left(j \phi_{i, m, k}^{[A]}+j \phi_{i, n, k}^{[D]}\right)}\right]^{T} \in C^{N \times 1}$ as the signal steering vector of the virtual array corresponding to the $i$ th path at the $k$ th pulse, where $N=N_{t} N_{r}$. Denote $A_{k}=$ $\left[\varphi_{1, k}, \ldots, \varphi_{I, k}\right], \rho_{k}=\left[\rho_{1, k}, \ldots, \rho_{I, k}\right]^{T}$, and $W_{k}=\left[w_{1,1, k}, \ldots\right.$, $\left.w_{N t, N r, k}\right]^{T}$, so the measurement radar data vector can be expressed as

$$
r_{k}=\left[r_{1,1, k}, \ldots, r_{N t, N t, k}\right]^{T}=A_{k} \rho_{k}+W_{k} .
$$

In order to be consistent with the motion model, the $k$ th pulse of the measurement data is expressed with the time $t$ corresponding to the $k$ th pulse.
TABLE 1: The parameters of the motion target.

\begin{tabular}{lcc}
\hline Parameter & Notation & Value \\
\hline Initial ground range & $x_{0}$ & $2000 \mathrm{~km}$ \\
Ionosphere height & $H$ & $350 \mathrm{~km}$ \\
Aircraft initial height & $z_{0}$ & $10 \mathrm{~km}$ \\
Maximum horizontal velocity & $v_{x}(0)$ & $500 \mathrm{~km} / \mathrm{h}$ \\
Maximum descending velocity & $v_{z \max }$ & $90 \mathrm{~km} / \mathrm{h}$ \\
Carrier frequency & $f_{C}$ & $20 \mathrm{MHz}$ \\
Impulse repetition frequency & $f_{S}$ & $50 \mathrm{~Hz}$ \\
\hline
\end{tabular}

2.2. Establish Maneuvering Target Model. In this paper, for generality, we consider a maneuvering aircraft which makes a $180^{\circ}$ circular turn in $T=30 \mathrm{~s}$ [10]. The motion parameters are listed in Table 1. All the multipath signals are considered to fall within the same range cell. The ground distance $x(t)$ and flight altitude of the target $z(t)$, respectively, are expressed as

$$
\begin{gathered}
x(t)=x(0)-\frac{v_{x}(0) T}{\pi} \sin \left(\frac{t \pi}{T}\right), \\
z(t)=z(0)+\frac{v_{z}(0) T}{\pi}\left(1-\cos \left(\frac{t \pi}{T}\right)\right) .
\end{gathered}
$$

The target tracks in two dimensions are shown in Figures 1 and 2 .

\section{Analysis of Micromultipath Effect Based on the Flat Earth Model}

3.1. Analysis of Micromultipath Effect. An OTH radar system considering the micromultipath effect based on the flat earth model is illustrated in Figure 3.

Since our purpose in this paper is to estimate the instantaneous height of the target, for simplicity and without loss of generality, we only consider the $2 \mathrm{D}$ position and velocity (range direction $x$ and altitude direction $z$ ). Then the target state at the time $t$ is expressed as

$$
\tilde{x}(t)=\left[\begin{array}{lllll}
x(t) & z(t) & v_{x}(t) & v_{z}(t)
\end{array}\right]^{T},
$$

where $v_{x}(t)$ and $v_{z}(t)$ are the velocity of the range direction and the altitude direction, respectively.

As the effect of the cross-range array apertures on the height estimation is small, only the effect of array apertures which lies in the range direction will be considered. Correspondingly both the transmit arrays and receive arrays are considered to be linear and located in the $x$-axis.

As the micromultipath effect exists, the combination of the transmit path and receive path has four components: Path I $\left(l_{1}: l_{1}\right)$, Path II $\left(l_{2}: l_{2}\right)$, Path III $\left(l_{1}: l_{2}\right)$, and Path IV $\left(l_{2}: l_{1}\right)$. Path III and Path IV are with the same length [6]. The slant range $l_{1}(t)$ and $l_{2}(t)$ is expressed with the distance $x(t)$, the height of the ionosphere $H$, and the target height $z(t)$ as

$$
\begin{aligned}
& l_{1}(t)=\sqrt{x(t)^{2}+(2 H-z(t))^{2}}, \\
& l_{2}(t)=\sqrt{x(t)^{2}+(2 H+z(t))^{2}} .
\end{aligned}
$$




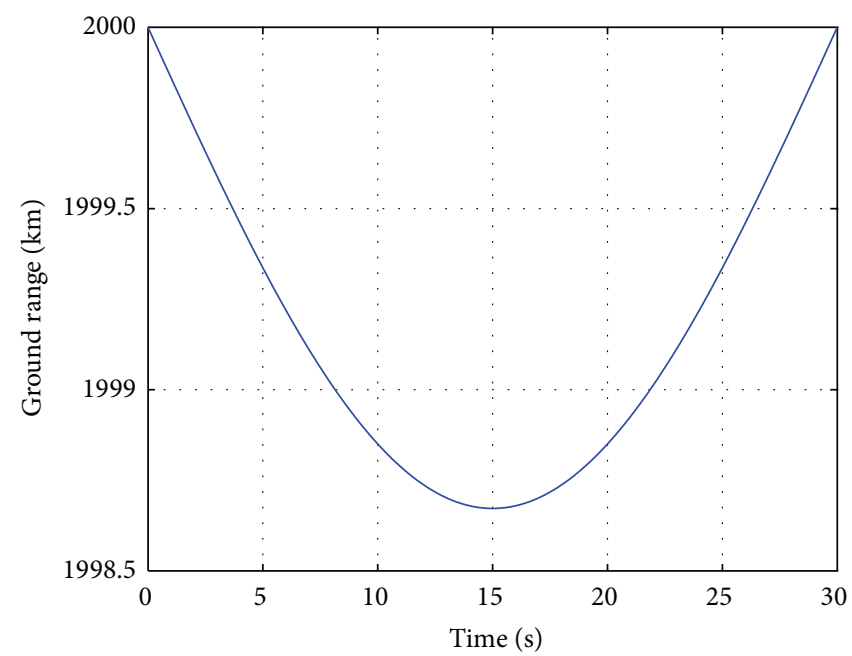

Figure 1: The target's track in the range direction.

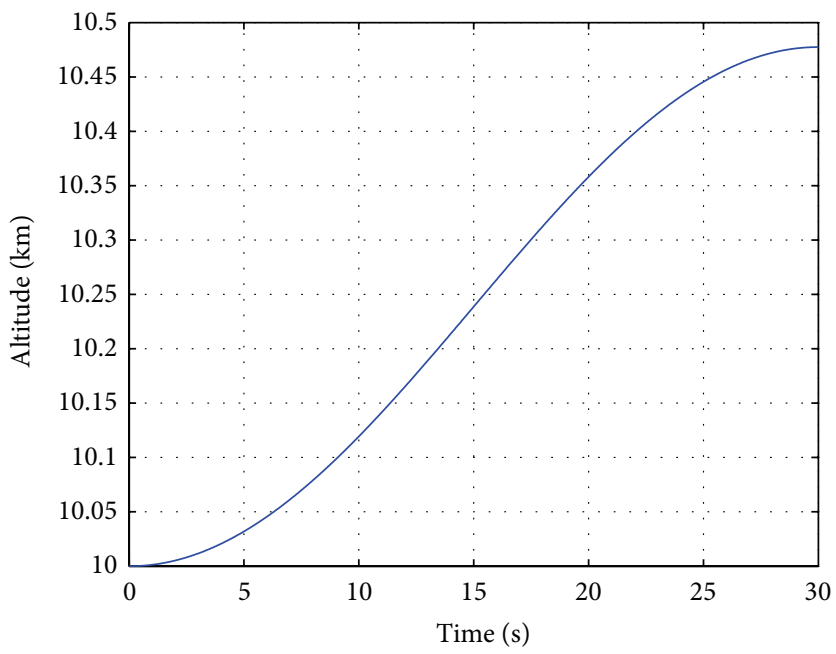

FIGURE 2: The target's track in the altitude direction.

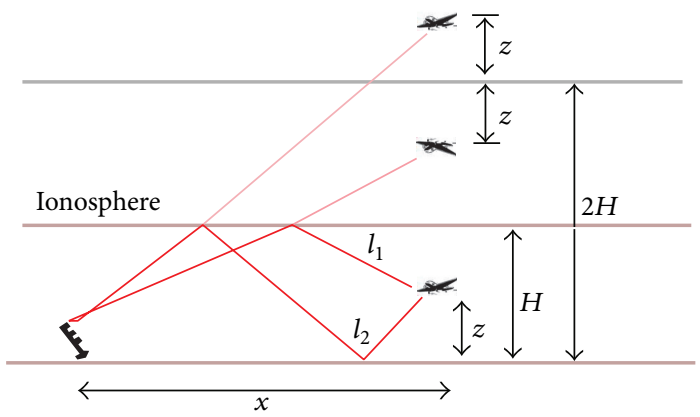

Figure 3: The micromultipath effect in the flat earth model. 
As $x \gg H \gg z, l_{1}(t)$ and $l_{2}(t)$ are simplified as

$$
\begin{aligned}
& l_{1}(t) \approx x(t)+\frac{2 H^{2}-2 H z(t)}{x(t)}, \\
& l_{2}(t) \approx x(t)+\frac{2 H^{2}+2 H z(t)}{x(t)} .
\end{aligned}
$$

Then $L_{1}(t)$ and $L_{2}(t)$, the length of Path I and Path II, are expressed as

$$
\begin{aligned}
& L_{1}(t)=2 l_{1}(t) \approx 2 x(t)+\frac{4 H^{2}-4 H z(t)}{x(t)}, \\
& L_{2}(t)=2 l_{2}(t) \approx 2 x(t)+\frac{4 H^{2}+4 H z(t)}{x(t)} .
\end{aligned}
$$

$L_{3}(t)$ and $L_{4}(t)$, the length of Path III and Path IV, are written as

$$
L_{3}(t)=L_{4}(t)=l_{1}(t)+l_{2}(t) \approx 2 x(t)+\frac{4 H^{2}}{x(t)} .
$$

3.2. Instantaneous Target Altitude Estimation. In (4), $\rho_{i}(t)$ comprises the targets' Doppler information. Here we express $\rho_{i}(t)$ as

$$
\rho_{i}(t)=\sigma_{i}(t) e^{-j 2 \pi L_{i}(t) / \lambda},
$$

where $i=1, \ldots, 4$. $i$ is the index of the path. $\lambda=c / f_{c}$ is the wavelength corresponding to carrier frequency $f_{c}$, and $\sigma_{i}(t)$ represents the combined effect of transmit power, target reflection, which is a function of radar cross-section (RCS), and ionospheric effect. $L_{i}(t)$ is the two-way slant range, and the expressions of $L_{i}(t)$ are shown in the previous section.

In general, the target's track consists of the movement in the range and altitude direction. This section will discuss the Doppler frequency of the target flight in two directions and then deduce the instantaneous expression of the target height.

Suppose that both the range between the target and radar and the height of the target are the functions of time. As the height of the ionosphere $H$ is changing slowly, we assume that $H$ is a constant value in the coherent time. So we can get

$$
\begin{aligned}
& \frac{d l_{1}(t)}{d t} \approx J(t) v_{x}(t)-\frac{2 H}{x(t)} v_{z}(t), \\
& \frac{d l_{2}(t)}{d t} \approx J(t) v_{x}(t)+\frac{2 H}{x(t)} v_{z}(t),
\end{aligned}
$$

where $J(t)=\left(1-2 H^{2} / x^{2}(t)\right), v_{x}(t)=d x(t) / d t$ is the target's velocity of the range direction, and $v_{z}(t)=d z(t) / d t$ is the target's velocity of the altitude direction. So the Doppler frequencies of the four paths are

$$
\begin{aligned}
& f_{1}(t)=\frac{2 f_{c}}{c} \frac{d l_{1}(t)}{d t} \approx \frac{2 f_{c}}{c} J(t) v_{x}(t)-\frac{4 f_{c} H}{x(t) c} v_{z}(t), \\
& f_{2}(t)=\frac{2 f_{c}}{c} \frac{d l_{2}(t)}{d t} \approx \frac{2 f_{c}}{c} J(t) v_{x}(t)+\frac{4 f_{c} H}{x(t) c} v_{z}(t), \\
& f_{3}(t)=f_{4}(t)=\frac{f_{c}}{c} \frac{d l_{1}(t)+d l_{2}(t)}{d t} \approx \frac{2 f_{c}}{c} J(t) v_{x}(t) .
\end{aligned}
$$

From (14)-(16), we find that the Doppler frequencies of the four paths have the same part $2 f_{c} J(t) v_{x}(t) / c$, which is determined by $v_{x}(t)$. We call this the dominant Doppler component. $c$ is the speed of light. $f_{1}$ and $f_{2}$ also have the part caused by $v_{z}(t)$. We call this the minor Doppler component, which contains the important information of the target movement in the altitude direction. Let $f_{2}(t)-f_{1}(t)$; then $v_{z}(t)$ is obtained by the expression

$$
v_{z}(t)=\frac{\left[f_{2}(t)-f_{1}(t)\right] x(t) c}{8 f_{c} H} .
$$

To get the target height, it is necessary to know the target initial height. In practice, we can get $L_{1}(t), L_{2}(t)$ and $L_{3}(t)$ by the time delay, and the transmit angle $\alpha$ and the ionospheric height $H$ can also be known by the other measurement equipment. Then these parameters can be used to calculate the initial height $z_{0}$. According to (8), we can get two estimated values $z_{0}$ by

$$
\begin{aligned}
& z_{0}=2 H-\sqrt{\left(\frac{L_{1}(0)}{2}\right)^{2}-x_{0}{ }^{2}}, \\
& z_{0}=\sqrt{\left(\frac{L_{2}(0)}{2}\right)^{2}+x_{0}{ }^{2}-2 H .}
\end{aligned}
$$

However, $z_{0}$ gotten by (18) is quite different from the actual value. It is because $z_{0}$ is very small compared to $x(t)$. If $x(t)$ is with a small error, there will be a great impact on $z_{0}$.

For elimination of the impact by the error of $x(t)$, we can use the following to calculate $z(t)$ :

$$
\begin{aligned}
& x(t)^{2}+[2 H-z(t)]^{2}=L_{1}{ }^{2}, \\
& x(t)^{2}+[2 H+z(t)]^{2}=L_{2}{ }^{2} .
\end{aligned}
$$

$z(t)$ is obtained by (19) as

$$
z(t)=\frac{L_{2}(t)^{2}-L_{1}(t)^{2}}{8 H} .
$$

Let $t=0, z_{0}$ be gotten. Then $z(t)$ can be written as

$$
z(t)=\frac{L_{2}(0)^{2}-L_{1}(0)^{2}}{8 H}+\frac{\left[f_{2}(t)-f_{1}(t)\right] x(t) c}{8 f_{c} H} \times t .
$$

\section{Analysis of Micromultipath Effect Based on Earth Curvature Model}

4.1. Analysis of Micromultipath Effect. In this section, the micromultipath effect model, which is under the influence of the earth curvature, will be discussed. The micromultipath effect model in an OTH radar system of the earth curvature model is illustrated in Figure 4. $x(t)$ is expressed as the ground range between the target and the radar. In order to be consistent with the definition in the flat earth model, $z(t)$ 


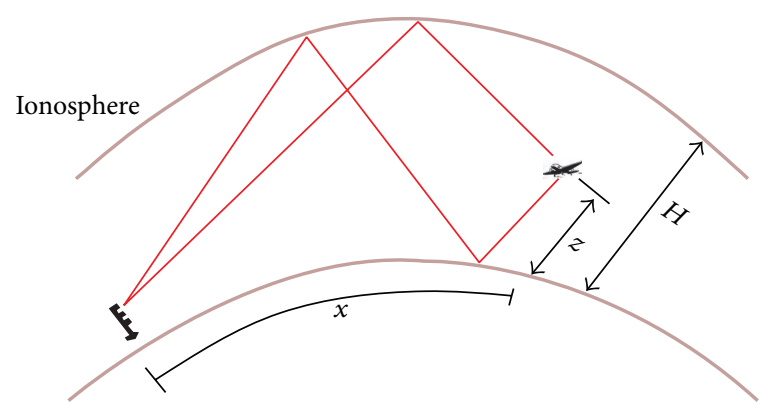

FIgURE 4: The micromultipath effect in the earth curvature model.

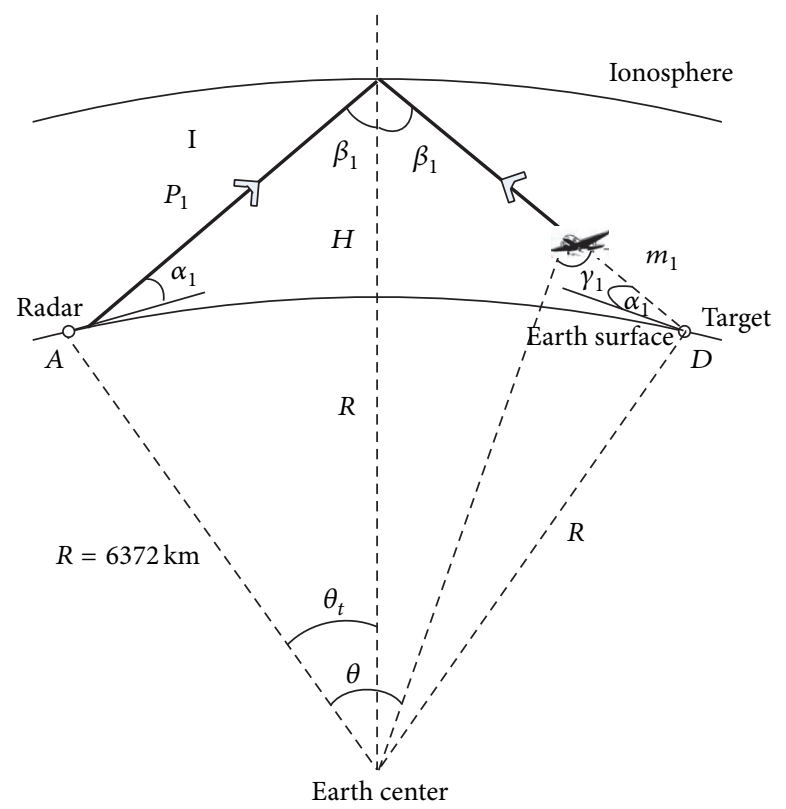

FIGURE 5: Path I in the earth curvature model.

indicates the vertical height, $H$ is the height of the ionosphere, and $R$ is the earth radius (it is set as a constant value in the paper; $R=6372 \mathrm{~km}$ ). Same as the flat earth model, the radar signal propagation paths in the earth curvature model have four combinations: Path I $\left(l_{1}: l_{1}\right)$, Path II $\left(l_{2}: l_{2}\right)$, Path III $\left(l_{1}: l_{2}\right)$, and Path IV $\left(l_{2}: l_{1}\right)$.

Path $\mathrm{I}$ is shown in Figure 5, in which $\alpha_{1}$ indicates the departure angle of radar transmitting antenna, $P_{1}$ is the distance of electromagnetic signal from radar transmitting antenna to ionosphere, $m_{1}$ is the extended line, and $\beta_{1}, \gamma_{1}$ indicate the auxiliary angles.

According to the geometrical relationship, $\alpha_{1}$ meets the following condition:

$$
\begin{gathered}
\frac{x(t)}{R}+\alpha_{1}+2 \arcsin \left[\frac{R \cos \left(\alpha_{1}\right)}{R+H}\right] \\
-\arcsin \left[\frac{R}{R+z} \cos \left(\alpha_{1}\right)\right]=\frac{\pi}{2} .
\end{gathered}
$$

The accurate value of $\alpha_{1}$ can be calculated by (22). The auxiliary angles $\beta_{1}$ and $\gamma_{1}$ can be expressed as the function of $\alpha_{1}$ :

$$
\beta_{1}=\arcsin \left[\frac{R \cos \left(\alpha_{1}\right)}{R+H}\right], \quad \gamma_{1}=\arcsin \left[\frac{R \cos \left(\alpha_{1}\right)}{R+z(t)}\right] \text {. }
$$

$P_{1}$ and $m_{1}$ can be, respectively, expressed as

$$
\begin{gathered}
P_{1}=\frac{R \cos \left(\alpha_{1}+\beta_{1}\right)}{\sin \beta_{1}}, \\
m_{1}=P_{1}+\frac{H+R}{\sin \gamma} \cos \left[\frac{x(t)}{R}+\alpha_{1}+\beta\right] .
\end{gathered}
$$

Then $l_{1}(t)$ is written as

$$
\begin{aligned}
l_{1}(t)=2 & P_{1}-m_{1}=R \frac{\cos \left(\alpha_{1}+\beta_{1}\right)}{\sin \beta_{1}} \\
& -\frac{H+R}{\sin \gamma_{1}} \cos \left[\frac{x(t)}{R}+\alpha_{1}+\beta_{1}\right] .
\end{aligned}
$$




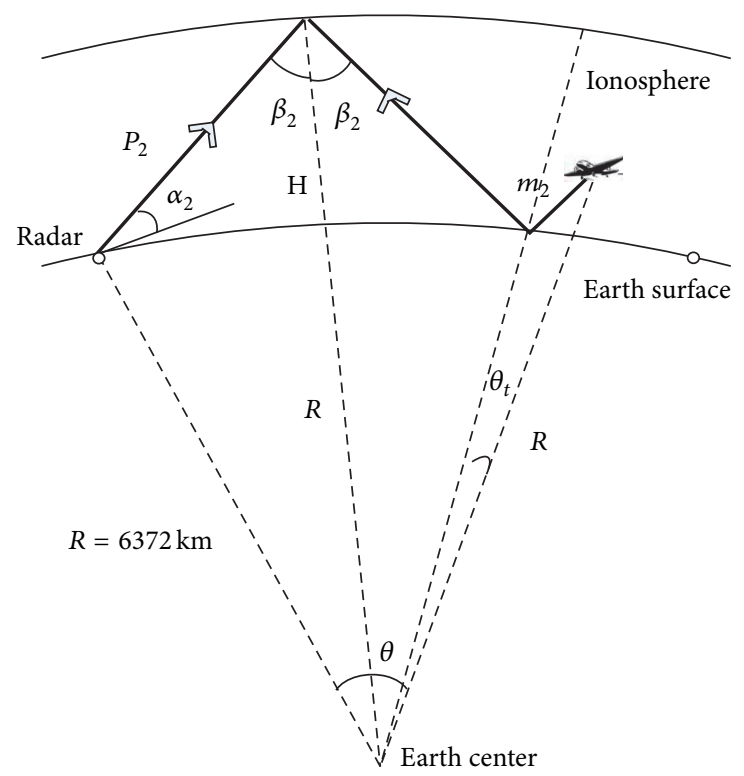

FIgURE 6: Path II in the earth curvature model.

Path II is illustrated in Figure 6. Similarly, $\alpha_{2}$ indicates the departure angle of radar transmitting antenna, $P_{2}$ is the distance of electromagnetic signal from radar transmitting antenna to ionosphere, $m_{2}$ is the extended line, and $\beta_{2}$ and $\gamma_{2}$ indicate the auxiliary angles.

From Figure 6, $\alpha_{2}$ should meet the following conditions:

$$
\begin{aligned}
& \frac{x(t)}{R}+\arcsin \left(\frac{R}{R+z} \cos \alpha_{2}\right) \\
& \quad+2 \arcsin \left(\frac{R}{R+H} \cos \alpha_{2}\right)+3 \alpha_{2}=\frac{3 \pi}{2} .
\end{aligned}
$$

Then $\alpha_{2}$ can be calculated by (26). The auxiliary angles $\beta_{2}$ and $\gamma_{2}$ can be expressed as the function of $\alpha_{2}$ :

$$
\beta_{2}=\arcsin \left(\frac{R \cos \alpha_{2}}{R+H}\right), \quad \gamma_{2}=\arcsin \left[\frac{R \cos \alpha_{2}}{R+z(t)}\right] .
$$

$P_{2}$ and $m_{2}$ can be, respectively, expressed as

$$
P_{2}=\frac{R \cos \left(\alpha_{2}+\beta_{2}\right)}{\sin \beta_{2}}, \quad m_{2}=\frac{\cos \left(\alpha_{2}+\gamma_{2}\right)}{\cos \alpha_{2}}[R+z(t)] .
$$

Then the expression of transmission oblique diameter $l_{2}(t)$ is as follows:

$$
\begin{array}{r}
l_{2}(t)=2 P_{2}+m_{2}=2 R \frac{\cos \left(\alpha_{2}+\beta_{2}\right)}{\sin \beta_{2}} \\
+\frac{\cos \left(\alpha_{2}+\gamma_{2}\right)}{\cos \alpha_{2}}[R+z(t)] .
\end{array}
$$

So $L_{1}(t)$ and $L_{2}(t)$, the length of Path I and Path II, are

$$
\begin{aligned}
L_{1}(t)= & 2 l_{1}(t)=2 R \frac{\cos \left(\alpha_{1}+\beta_{1}\right)}{\sin \beta_{1}} \\
& -2 \frac{H+R}{\sin \gamma_{1}} \cos \left[\frac{x(t)}{R}+\alpha_{1}+\beta_{1}\right],
\end{aligned}
$$

$$
L_{2}(t)=2 l_{2}(t)=4 R \frac{\cos \left(\alpha_{2}+\beta_{2}\right)}{\sin \beta_{2}}
$$

And $L_{3}(t)$ and $L_{4}(t)$, the length of Path III and Path IV, are shown as

$$
+2 \frac{\cos \left(\alpha_{2}+\beta_{2}\right)}{\cos \alpha_{2}}[R+z(t)]
$$

$$
\begin{aligned}
L_{3}(t)= & L_{4}(t)=l_{1}(t)+l_{2}(t) \\
= & R \frac{\cos \left(\alpha_{1}+\beta_{1}\right)}{\sin \beta_{1}}-\frac{H+R}{\sin \gamma_{1}} \cos \left[\frac{x(t)}{R}+\alpha_{1}+\beta_{1}\right] \\
& +2 R \frac{\cos \left(\alpha_{2}+\beta_{2}\right)}{\sin \beta_{2}}+\frac{\cos \left(\alpha_{2}+\gamma_{2}\right)}{\cos \alpha_{2}}[R+z(t)]
\end{aligned}
$$

4.2. Instantaneous Target Altitude Estimation. Consistent with the processing methods in the flat earth model, we 


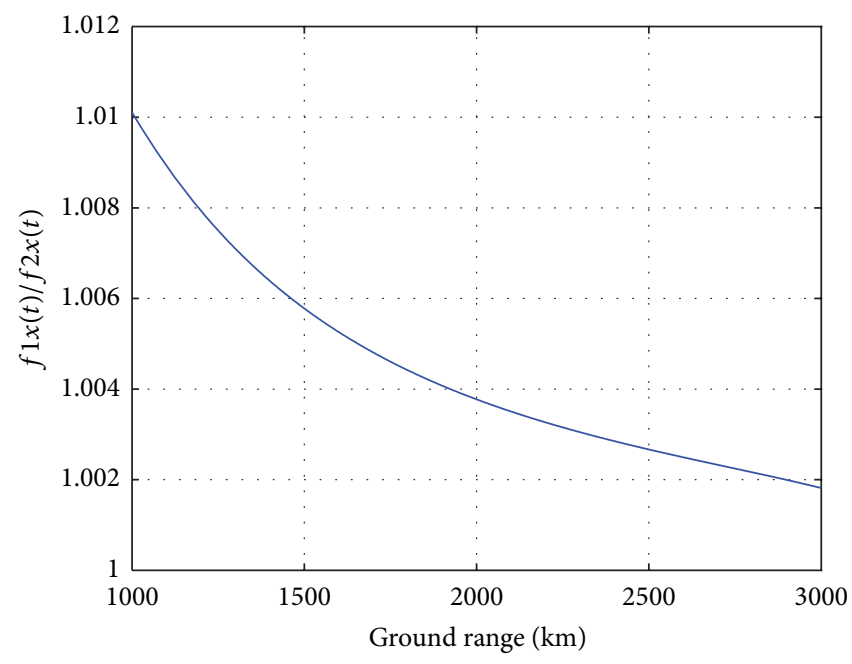

Figure 7: The ration of $11 \mathrm{x}$ to $12 \mathrm{x}$.

suppose that $H$ is constant in the coherent time. Then the time derivatives of the $l_{1}(t), l_{2}(t), l_{3}(t)$, and $l_{4}(t)$ are obtained by

$$
\begin{aligned}
& \frac{d l_{1}(t)}{d t}=\frac{\sin \left[\alpha_{1}+\beta_{1}+x(t) / R\right][R+z(t)](H+R)}{R^{2} \cos \alpha_{1}} v_{x}(t) \\
& -\frac{\cos \left[\alpha_{2} / 2+\beta_{1}+x(t) / R\right](H+R)}{R \cos \alpha_{1}} v_{z}(t) \\
& =l_{1 x} v_{x}(t)+l_{1 z} v_{z}(t) \text {, } \\
& \frac{d l_{2}(t)}{d t}=\left(\left(\cos \left[\frac{\alpha_{2}}{2}-\frac{\pi}{4}+\frac{\gamma}{2}+\frac{x(t)}{2 R}\right](H+R)\right)\right. \\
& \left.\times\left(R \cos \alpha_{2}\right)^{-1}\right) v_{x}(t) \\
& +\left\{\frac{\cos \left(\alpha_{2}+\gamma_{2}\right)}{\cos \alpha_{2}}+\frac{R \sin \left(\alpha_{2}+\gamma_{2}\right)}{[R+z(t)] \cos \gamma_{2}}\right. \\
& -\left(R \cos \left[\frac{\alpha_{2}}{2}-\frac{\pi}{4}+\frac{\gamma}{2}+\frac{x(t)}{2 R}\right](H+R)\right) \\
& \left.\times\left(\sqrt{1-\left[\frac{R \cos \alpha_{2}}{R+z(t)}\right]^{2}}[R+z(t)]^{2}\right)^{-1}\right\} v_{z}(t), \\
& =l_{2 x} v_{x}(t)+l_{2 z} v_{z}(t) \\
& \frac{d l_{3}(t)}{d t}=\frac{d l_{4}(t)}{d t}=\frac{1}{2}\left[\frac{d l_{1}(t)}{d t}+\frac{d l_{2}(t)}{d t}\right] .
\end{aligned}
$$

$l_{1 x}$ is the coefficient of the part of $d l_{1}(t) / d t$ caused by $v_{x}(t) . l_{1 z}$ is the coefficient of the part of $d l_{1}(t) / d t$ caused by $v_{z}(t)$. The expressions of $l_{2 x}$ and $l_{2 z}$ are similar to $l_{1 x}$ and $l_{1 z}$, but replace $l_{1}$ with $l_{2}$. So the Doppler frequencies produced by the moving target of the four paths can be, respectively, written as

$$
\begin{aligned}
f_{1}(t) & =\frac{2 f_{c}}{c} \frac{d l_{1}(t)}{d t}, \\
f_{2}(t) & =\frac{2 f_{c}}{c} \frac{d l_{2}(t)}{d t}, \\
f_{3}(t)=f_{4}(t) & =\frac{1}{2}\left[f_{1}(t)+f_{2}(t)\right] .
\end{aligned}
$$

The four Doppler frequencies both consist of the components caused by $v_{x}(t)$ and $v_{z}(t)$. The ratio of $l_{1 x}$ to $l_{2 x}$ under the condition that $x(t)$ is made to change from $1000 \mathrm{~km}$ to $3000 \mathrm{~km}$ is shown in Figure 7. From the figure, it can be found that the values of Doppler frequency components caused by $v_{x}(t)$ in $f_{1}(t)$ and $f_{2}(t)$ are very close. And it indicates that the Doppler frequency component in each path caused by $v_{x}(t)$ is approximately equal. This conclusion is same as the one in the flat earth model. Therefore, $v_{z}(t)$ can be obtained by $f_{2}(t)-f_{1}(t)$ as

$$
v_{z}(t)=\frac{c}{2 f_{c}} \frac{f_{2}(t)-f_{1}(t)}{l_{2 z}-l_{1 z}} .
$$

To get the instantaneous height of the moving target, the initial height of the target $z_{0}$ is needed. Different from the situation in the flat earth model, $z_{0}$ can be calculated accurately by using $L_{1}(t)$ or $L_{2}(t)$. In order to reduce random errors, $L_{1}(t)$ and $L_{2}(t)$ are used to calculate the initial heights $z_{01}, z_{02}$ and then make the average of $z_{01}, z_{02}$ as $z_{0}$.

For Path I, $z_{01}$ can be shown as follows:

$$
z_{01}=\left[\frac{L_{1}(0)}{2}-\frac{\sin \theta_{1}}{\sin \beta_{1}}\right] \frac{\sin \beta_{1}}{\sin \theta_{2}}-R,
$$

where

$$
\begin{aligned}
& \beta_{1}=\arcsin \left(\frac{R \cos \alpha_{1}}{H+R}\right), \\
& \theta_{2}=\frac{x(t)}{R}-\frac{\pi}{2}+\alpha_{1}+\beta_{1} .
\end{aligned}
$$




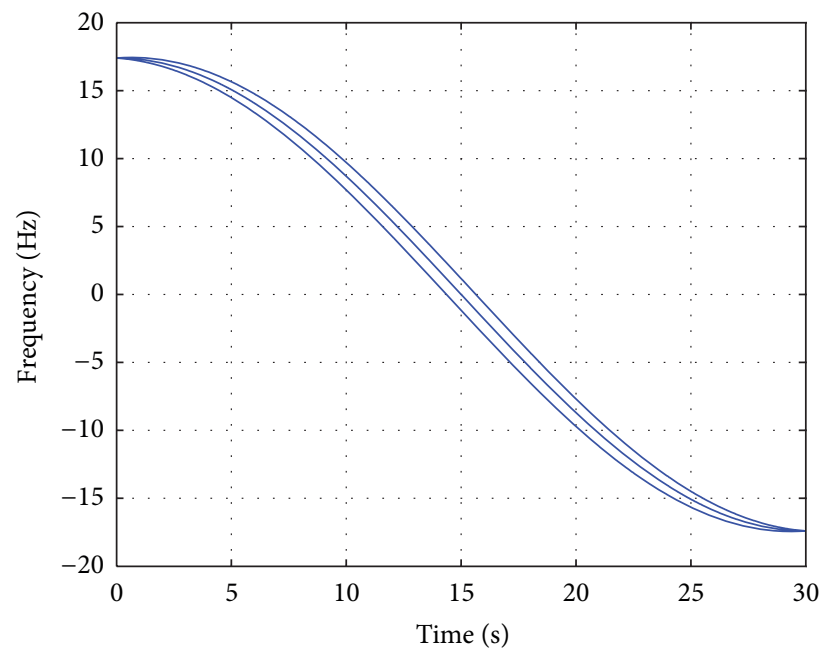

FIGURE 8: The Doppler frequency curves of the moving target in the flat earth model.

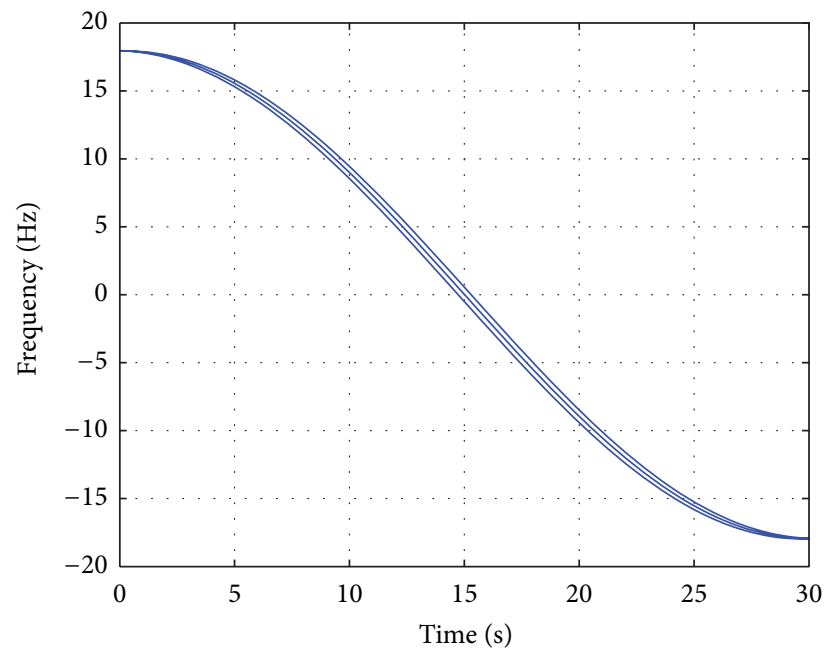

Figure 9: The Doppler frequency curves of the moving target in the earth curvature model.

For Path II, $z_{02}$ can be written as

$$
z_{02}=\frac{R \cos \alpha_{2}}{\sin \gamma_{0}}-R
$$

where

$$
\begin{aligned}
& \gamma_{2}=\frac{3 \pi}{2}-\frac{x(t)}{R}-2 \alpha_{2}-2 \beta_{2}, \\
& \beta_{2}=\arcsin \left(\frac{R}{R+H}\right) \times \cos \alpha_{2} .
\end{aligned}
$$

Then $z_{0}$ is the average value of $z_{01}$ and $z_{02}$ :

$$
\begin{aligned}
z_{0}= & \left(\left[\frac{L_{1}(0)}{2}-\frac{\sin \theta_{1}}{\sin \beta_{1}}\right] \frac{\sin \beta_{1}}{\sin \theta_{2}}+\frac{R \cos \alpha_{2}}{\sin \gamma_{0}}-2 R\right) \\
& \times(2)^{-1} .
\end{aligned}
$$

So the instantaneous height $z(t)$ can be expressed as

$$
z(t)=z_{0}+v_{z}(t) \times t .
$$

\section{Comparison of the Difference of Micromultipath Effects between Two Kinds of Models}

The aim of this section is to analyze and compare the difference of micromultipath effects between the flat earth model and the earth curvature model. The moving target model established in Section 2.2 is used in this section.

5.1. The Difference in the Doppler Frequencies Produced by the Target's Movement. The curves of Doppler frequencies of the four paths produced by the target's movement in the flat earth model and the earth curvature model are shown in Figure 8 and Figure 9, respectively. As can be seen from the figures, 


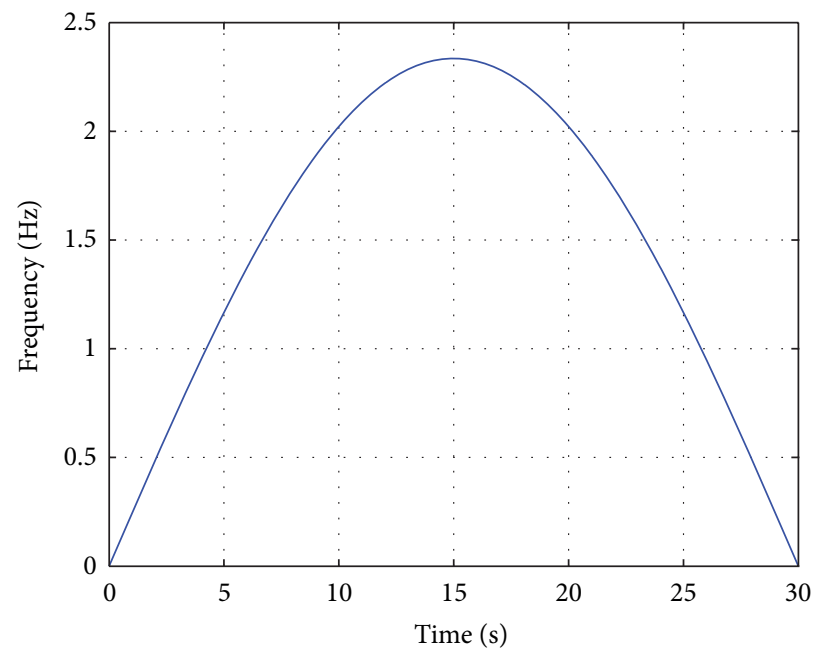

Figure 10: The Doppler frequency difference between Path I and Path II in the flat earth model.

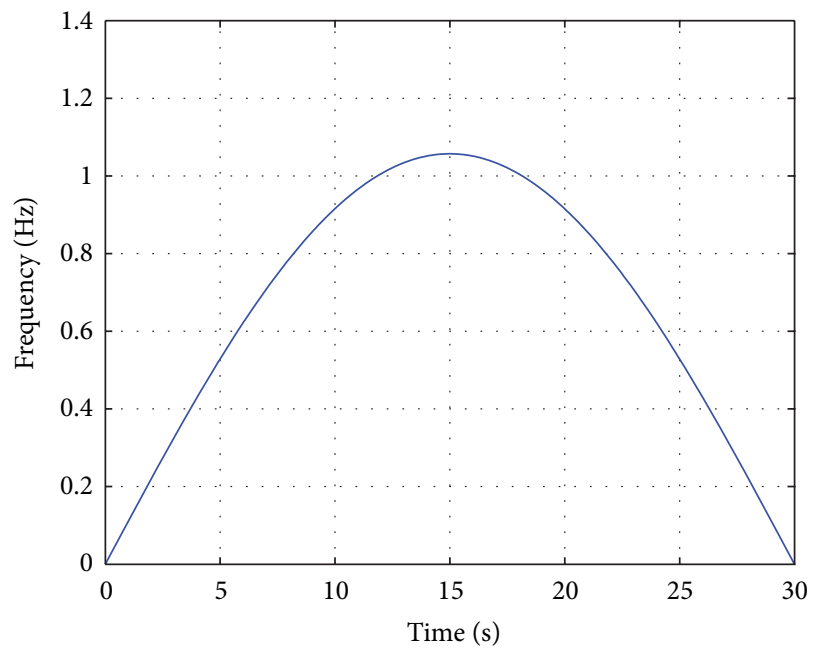

Figure 11: The Doppler frequency difference between Path I and Path II in the earth curvature model.

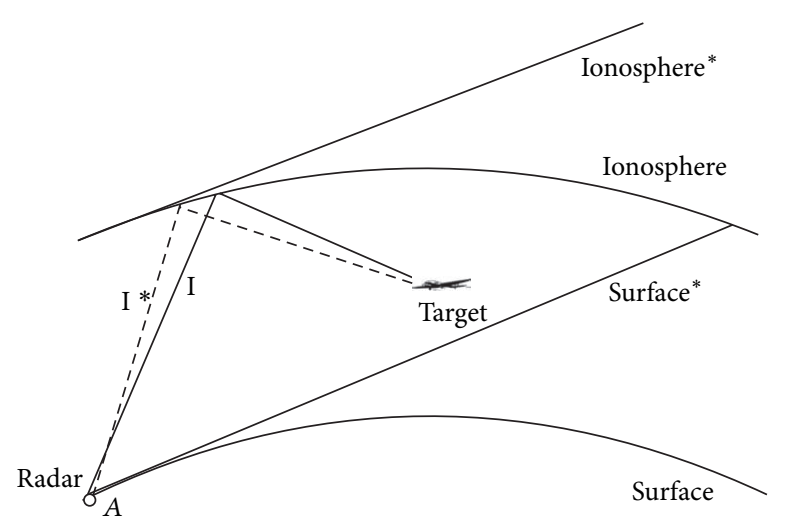

(a) Path I

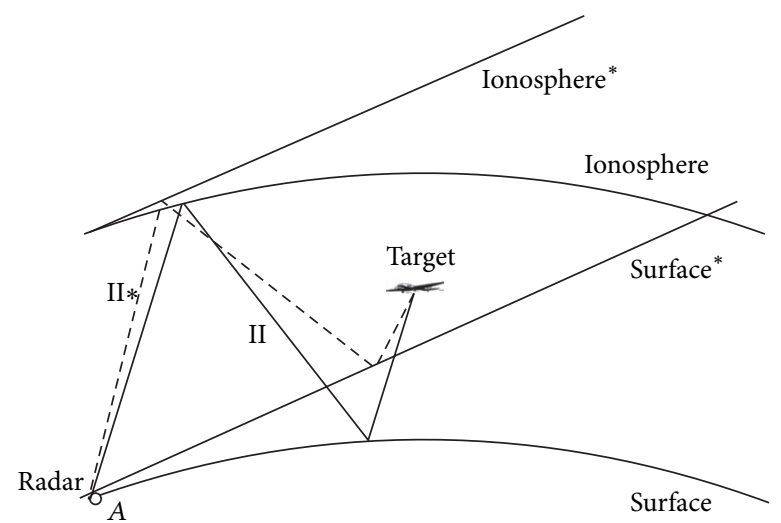

(b) Path II

Figure 12: The Path I and Path II in two kinds of models. 


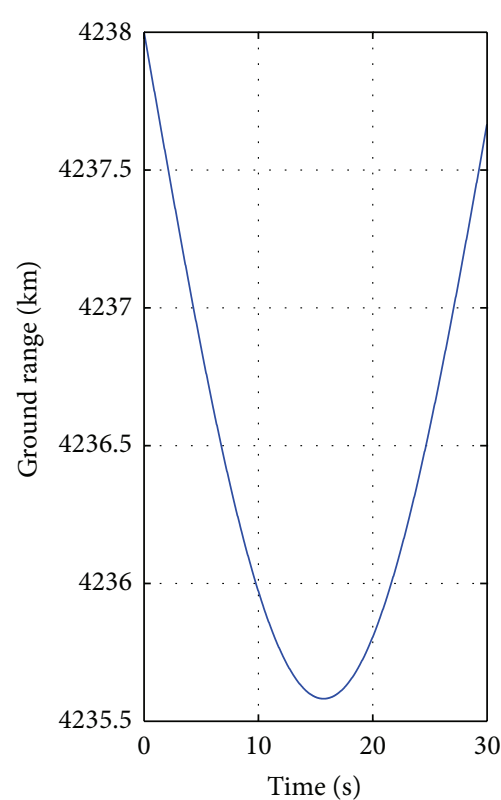

(a) Path I in the flat earth model

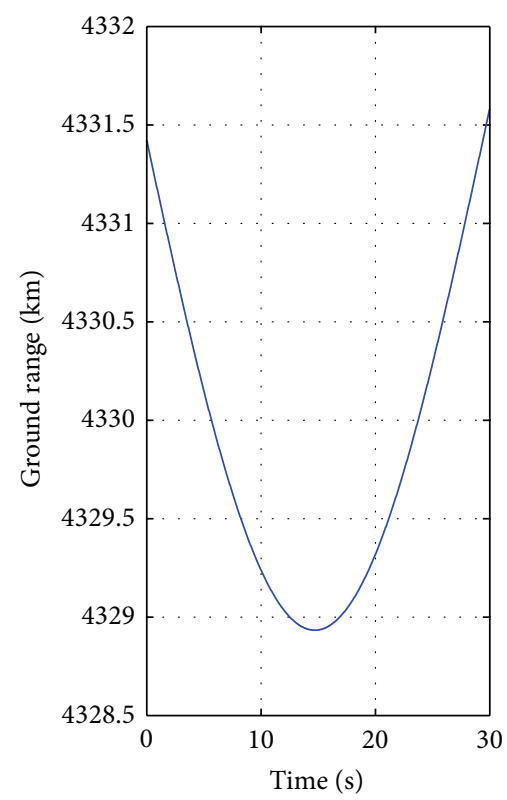

(c) Path I in the earth curvature model

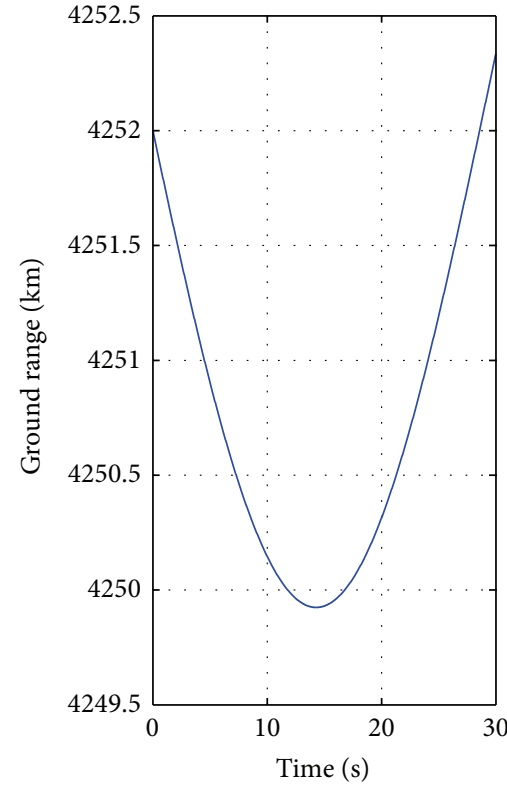

(b) Path II in the flat earth model

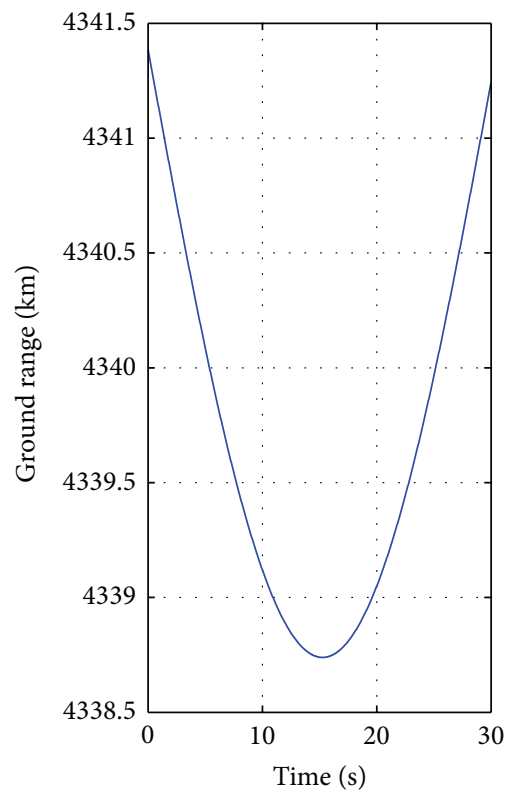

(d) Path II in the earth curvature model

FIgURE 13: The simulation results of Path I and Path II in two kinds of models.

the target turns an angle of 180 degrees on the ground, so the value of Doppler frequency reduces from the maximum to the minimum. At the start and the end points of the movement period, for the altitude velocities are both zero, the Doppler frequency of each path is the same. For the absolute value of $v_{x}(t)$ is maximal, the absolute value of Doppler frequency comes to the maximum.

At the moment $T / 2, v_{x}(t)$ comes to zero, and the Doppler frequencies of every path are close to zero, while $v_{z}(t)$ reaches its maximum. Therefore, the Doppler frequencies of every path come to its maximum difference value at this moment, which is the best moment to obtain the Doppler frequency differences produced by $v_{z}(t)$.

By comparison of Figures 8 and 9, the Doppler frequency differences of the every path in the earth curvature model are smaller than the corresponding results in the flat earth model. Figures 10 and 11 illustrate, respectively, the values of the Doppler frequency differences between Path I and Path II in the flat earth model and the earth curvature model within one movement period. As seen, the maximum value of Doppler frequency difference in the flat earth model is $2.4 \mathrm{~Hz}$ and the one in the earth curvature model only is $1.3 \mathrm{~Hz}$. It indicates 


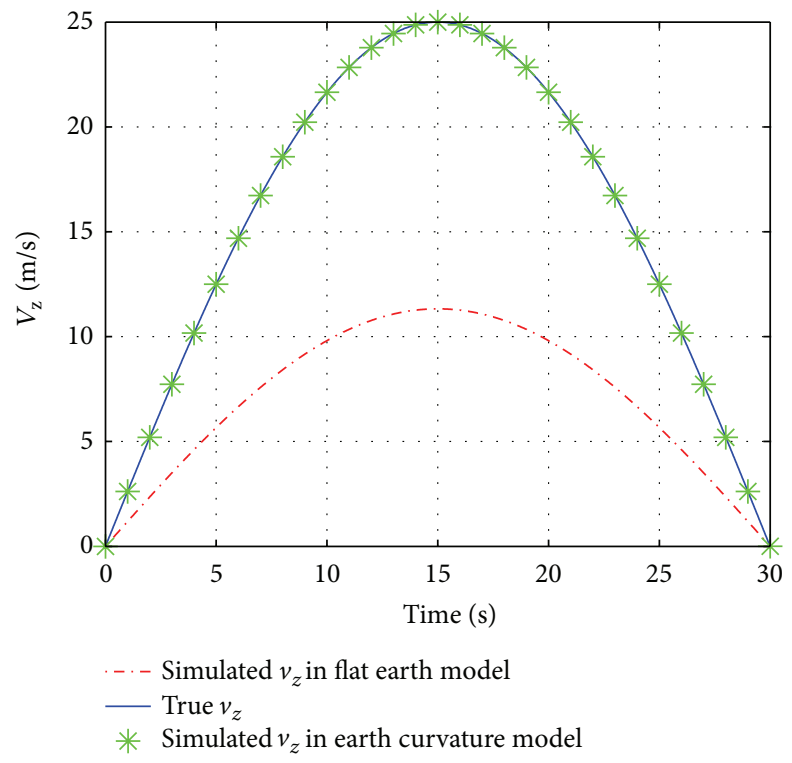

FIGURE 14: The comparison of $v_{z}$ calculated by two kinds of earth models.

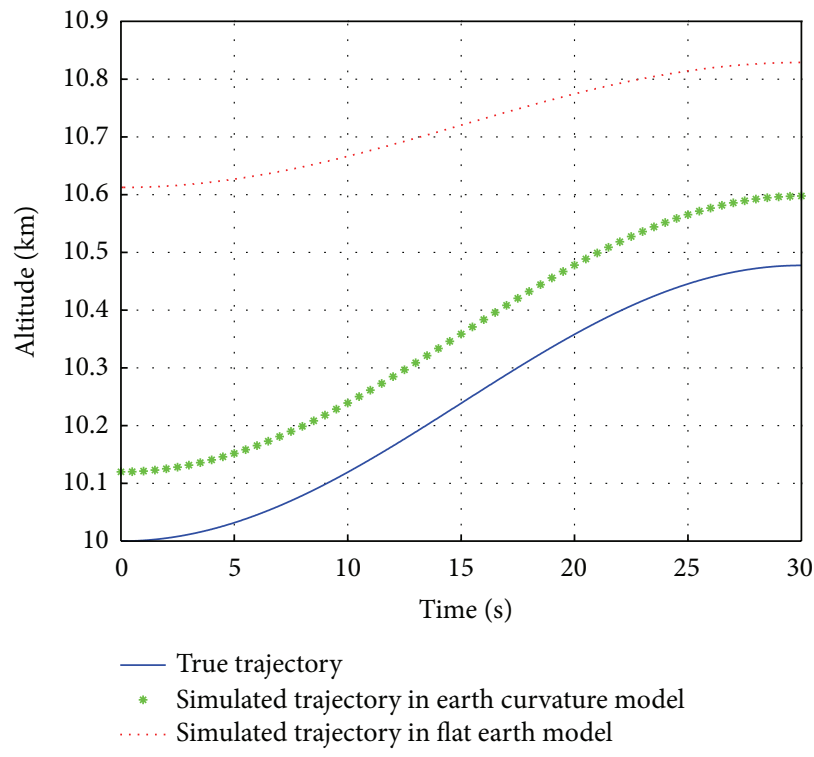

FIGURE 15: The comparison of the movement tracks calculated in two kinds of earth models.

that the error of the Doppler frequency of the flat earth model is close to $1.1 \mathrm{~Hz}$, which will bring larger error in the altitude estimation.

5.2. The Difference in the Target Track. Figures 12(a) and 12(b) illustrate Path I and Path II in two kinds of models, in which the solid line represents the propagation path in the earth curvature model and the dotted line represents the propagation path in the flat earth model. The superscript $*$ denotes the reference planes in the flat earth model. From these figures, we cannot determine directly in which model the propagation path is longer. So the tracks within one movement period are shown in Figure 13. In this figure, the varied tendencies of the signal propagation path in two kinds of models are basically identical, the length of Path I in the earth curvature model is about $94 \mathrm{~km}$ longer than it is in the flat earth model, and the length of Path II in the earth curvature model is about $89 \mathrm{~km}$ longer than it is in the flat earth model.

The Doppler frequency difference $f_{\text {diff }}$, between Path I and Path II in the flat earth model, can be obtained by (14) and (15) as

$$
f_{\text {diff }}=\frac{8 f_{c} H}{x(t) c} v_{z}(t)
$$

From (41), $f_{\text {diff }}$ is inversely proportional to $x(t)$. For the signal propagation path in the earth curvature model is 


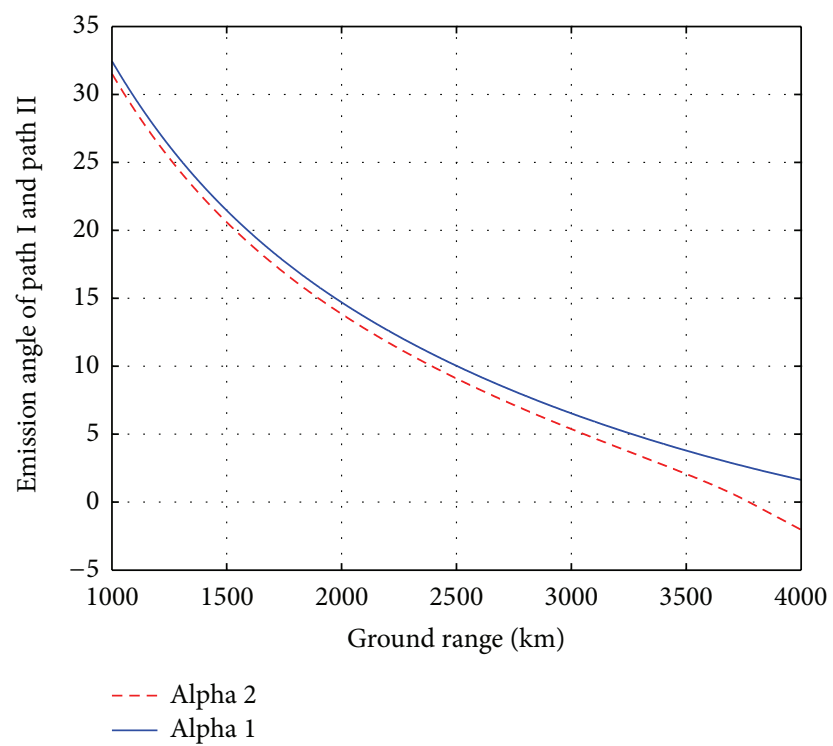

FIGURE 16: The curve of the emission angles $\alpha_{1}$ and $\alpha_{2}$ changing with the ground distance.

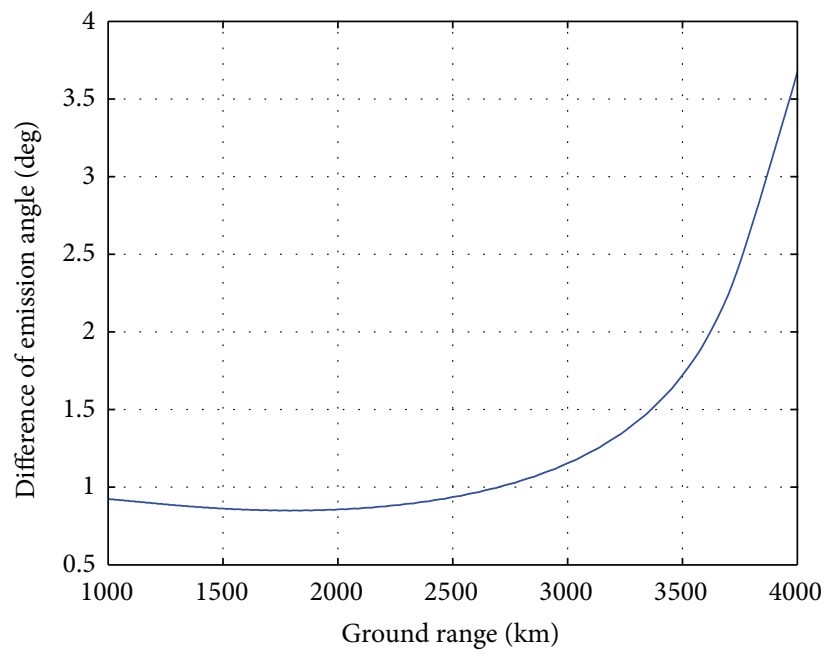

FIgURE 17: The curve of the difference $\alpha_{\text {diff }}$ changing with $x(t)$.

longer than the one in the flat earth model; $x(t)$ in the earth curvature model is larger than the one in the flat earth model. This is why the Doppler frequency differences of each path in the earth curvature model are smaller than the one in the flat earth model.

Figure 14 illustrates the comparison of the measured values $\widehat{v}_{z}(t)$ of the height velocity in two kinds of earth models with its actual value $v_{z}(t)$. It can be seen that $\widehat{v}_{z}(t)$ calculated in the earth curvature model is identical to the actual value, and $\widehat{v}_{z}(t)$ obtained in the flat earth model has great differences from the actual one. The error of $\widehat{v}_{z}(t)$ comes to $12 \mathrm{~m} / \mathrm{s}$ when $v_{z}(t)$ is with the maximum value, so the altitude estimation would have great bias if the flat earth model is adopted.

Figure 15 shows the track in the altitude direction with two kinds of models. In the earth curvature model, $\widehat{v}_{z}(t)$ has no error, so the varied tendency of the calculated track (the dotted line) coincides with the actual one (the real line). The initial height $z_{0}$ calculated by (39) has a slight bias about $120 \mathrm{~m}$. So the whole track has a fixed error with the true track. While $\widehat{v}_{z}(t)$ and $z_{0}$ calculated in the flat earth model both have a larger error, the varied tendency of the whole movement track and the altitude value at every moment have great difference from the real value.

\subsection{The Application Condition of the Earth Curvature Model}

5.3.1. The Requirement of the Skywave Radar Altimetry to the Transmission Antenna Beam Width $\Omega$. In order to use the effect of micromultipath, the transmission beam is requested to have a certain width to receive the echoes of four paths. Therefore, the transmission antenna beam width $\Omega$ must be larger than two times of the difference $\alpha_{\text {diff }}$ between the 


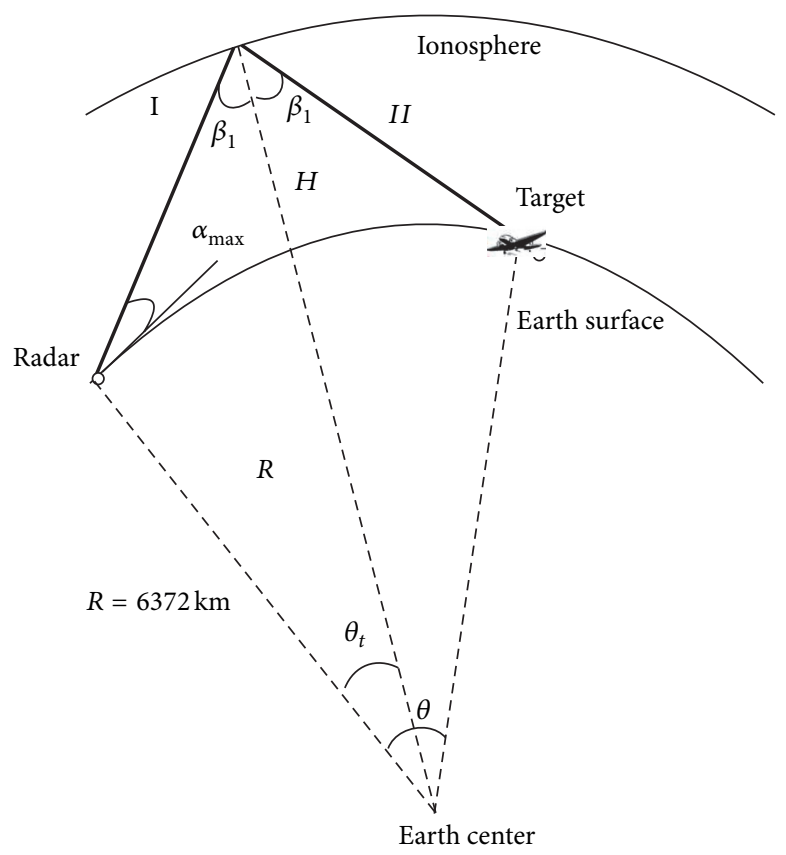

FIgURE 18: The illustration of the minimum detection range of the skywave radar.

emission angle $\alpha_{1}$ and $\alpha_{2}$ corresponding to Path I and Path II. In Figure 16, the changing curves of the emission angle $\alpha_{1}$ (the real line) and $\alpha_{2}$ (the dotted line) corresponding to Path I and Path II are shown when the ground distance $x(t)$ increases from $1000 \mathrm{~km}$ to $4000 \mathrm{~km}$. Figure 17 illustrates the curve of the difference $\alpha_{\text {diff }}$ between the emission angles of two paths changing with $x(t)$. We can find that the difference $\alpha_{\text {diff }}$ between the emission angles corresponding to Path I and Path II changes little (its biggest value is about $1.2^{\circ}$ ) within the detection range from $1000 \mathrm{~km}$ to $3000 \mathrm{~km}$. When the detection range exceeds $3000 \mathrm{~km}, \alpha_{\text {diff }}$ rises sharply, and it reaches about $3.7^{\circ}$ when the detection range is $4000 \mathrm{~km}$. Accordingly, when the skywave radar use micromultipath effect to measure the height, its beam width needs to be larger than $2.4^{\circ}$ if the detection range is smaller $3000 \mathrm{~km}$ and the beam width of the transmission antenna has to be larger than $7.4^{\circ}$ when the detection range exceeds $3000 \mathrm{~km}$.

5.3.2. The Detection Range of the Earth Curvature Model. The detection range of the skywave radar is limited in practice, for the emission angle $\alpha$ can only change in a certain range. The maximum value and the minimum value of $\alpha$ are written, respectively, as $\alpha_{\max }$ and $\alpha_{\min }$.

When the target's altitude is close to the ground and the emission angle is $\alpha_{\max }$, the sky wave radar can realize the detection of the minimum range, as shown in Figure 18. When the emission angle is $\alpha_{\min }$, the maximum detection is obtained, as shown in Figure 19.

The emission angle $\alpha_{\max }$ is generally taken as $30^{\circ}$. Then the minimum detection range $x_{\min }$ can be derived as follows:

$$
x_{\min }=\frac{2 \pi R}{3}-2 R \arcsin \left(\frac{\sqrt{3} R}{2(R+H)}\right) .
$$

The emission angle $\alpha_{\min }$ is always $0^{\circ}$; then the maximum detection range $x_{\max }$ can be derived as

$$
x_{\max }=\pi R-2 R \arcsin \left(\frac{R}{R+H}\right) .
$$

Taking the earth radius as $R=6372 \mathrm{~km}$ and the ionosphere height as $H=350 \mathrm{~km}$, then $x_{\min }=1072 \mathrm{~km}$ and $x_{\max }=4131 \mathrm{~km}$. The results conform to the practical detection range of the skywave radar.

\section{Conclusion}

Skywave OTH radar systems can make use of the micromultipath effect to estimate the instantaneous altitude of maneuvering targets. The flat earth model is often used. However, ignoring the impact of the curvature of the earth, the analytical expressions of instantaneous target altitude are relatively simple, making it suitable for descriptive analysis. And the initial altitude has a great difference from actual altitude (600 meters) in the flat earth model. Moreover, the curvature of the earth should not be ignored for the thousands of kilometers' detection in the skywave radar.

This paper focuses on the theoretical derivations about how to introduce the earth curvature model into the estimation of the target's altitude. The expressions of the Doppler frequency in four paths are deduced. And the difference of two kinds of models is compared. Although the analytical expressions are quiet complicated, the estimation of the initial altitude is close to the actual one. Therefore, any altimetry methods that are based on real data can improve the estimation results by exploiting the altimetry correction factors derived from earth curvature model. 


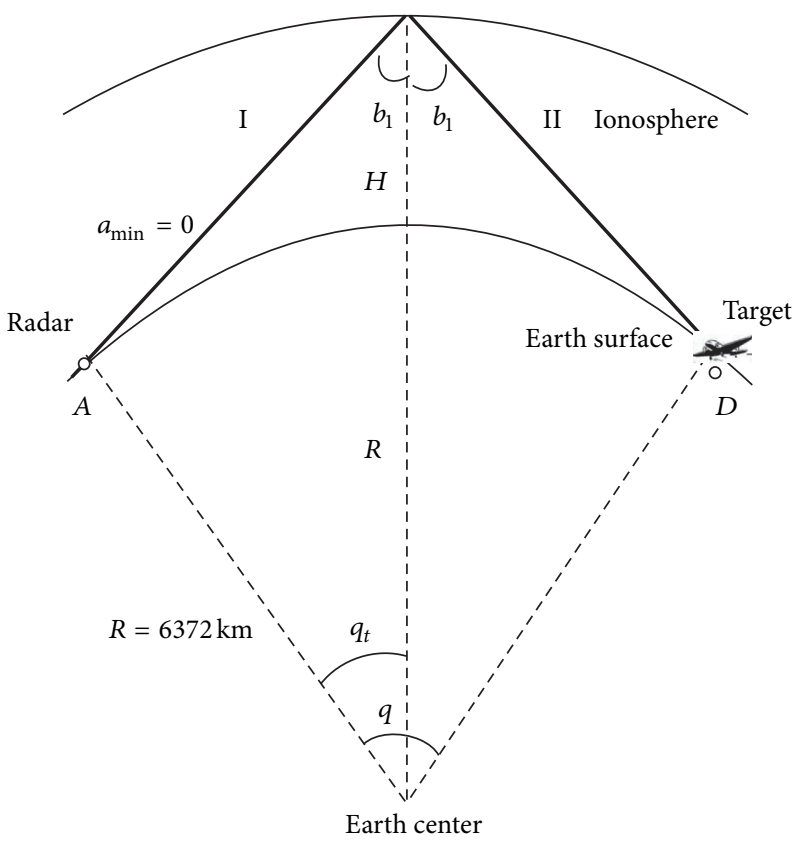

FIGURE 19: The illustration of the maximum detection range of the skywave radar.

\section{Conflict of Interests}

The authors declare that there is no conflict of interests regarding the publication of this paper.

\section{Acknowledgments}

At the point of finishing this paper, the authors would like to express their sincere thanks to the National Natural Science Foundation (no. 61201303) and the Fundamental Research Funds for the Central Universities (HIT.NSRIF.2013027).

\section{References}

[1] P. Jiang and J. Chen, "Research of OTH sky wave radar height finding based on matching domain processing," Journal of Air Force Radar Academy, pp. 157-165, 2012.

[2] X. Guo, J. Ni, W. Su, and G. Liu, "Development of sky wave overthe-horizon radar," Acta Aeronautica et Astronautica Sinica, vol. 23, no. 6, pp. 495-500, 2002.

[3] W. C. Anderson, D. S. Green, and S. P. Kingsley, "HF skywave radar: estimating aircraft heights using super-resolution in range," IEE Proceedings on Radar, Sonar and Navigation, vol. 143, no. 4, pp. 281-285, 1996.

[4] R. H. Anderson, S. Kraut, and J. L. Krolik, "Robust altitude estimation for over-the-horizon radar using a state-space multipath fading model," IEEE Transactions on Aerospace and Electronic Systems, vol. 39, no. 1, pp. 192-201, 2003.

[5] J. Praschifka, L. J. Durbridge, and J. Lane, "Investigation of target altitude estimation in skywave OTH radar using a high-resolution ionospheric sounder," in Proceedings of the International Radar Conference on Surveillance for a Safer World (RADAR '09), pp. 1-6, IEEE, Bordeaux, France, October 2009.

[6] M. Papazoglou and J. L. Krolik, "Electromagnetic matchedfield processing for target height finding with over-the-horizon radar," in Proceedings of the IEEE International Conference on Acoustics, Speech, and Signal Processing (ICASSP '97), pp. 559562, April 1997.

[7] M. Papazoglou and J. L. Krolik, "Matched-field estimation of aircraft altitude from multiple over-the-horizon radar revisits," IEEE Transactions on Signal Processing, vol. 47, no. 4, pp. 966976, 1999.

[8] M. Papazoglou and J. L. Krolik, "Electromagnetic matchedfield processing for target height finding with over-the-horizon radar," in Proceedings of the IEEE International Conference on Acoustics, Speech, and Signal Processing, (ICASSP '97), pp. 559562, April 1997.

[9] Y. D. Zhang, M. G. Amin, and B. Himed, "Altitude estimation of maneuvering targets in MIMO Over-the-horizon-radar," in Proceedings of the IEEE 7th Sensor Array and Multichannel Signal Processing Workshop, pp. 257-230, 2012.

[10] Y. Zhang, M. G. Amin, and G. J. Frazer, "High-resolution timefrequency distributions for manoeuvring target detection in over-the-horizon radars," IEE Proceedings: Radar, Sonar and Navigation, vol. 150, no. 4, pp. 299-304, 2003. 


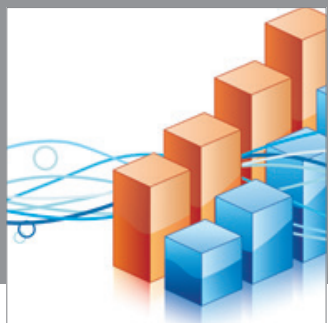

Advances in

Operations Research

mansans

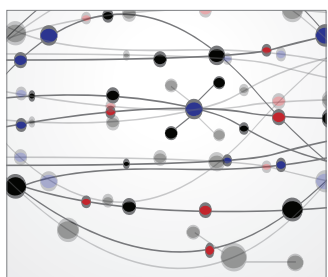

The Scientific World Journal
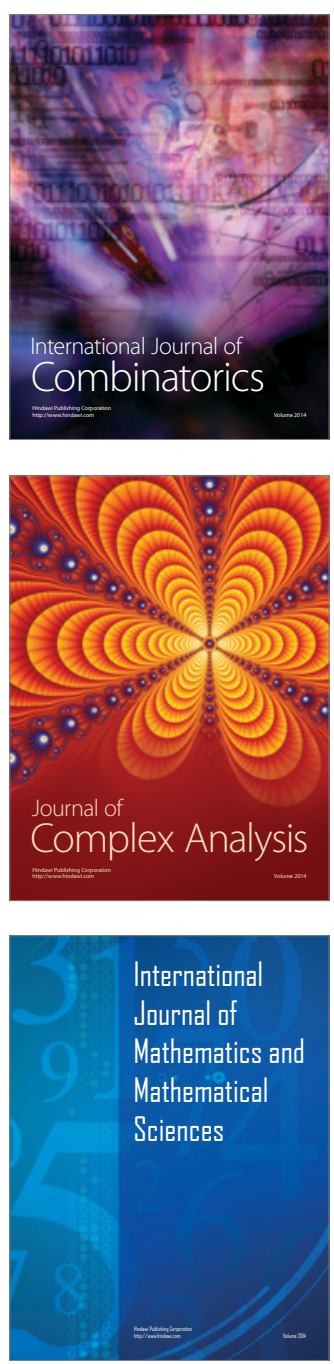
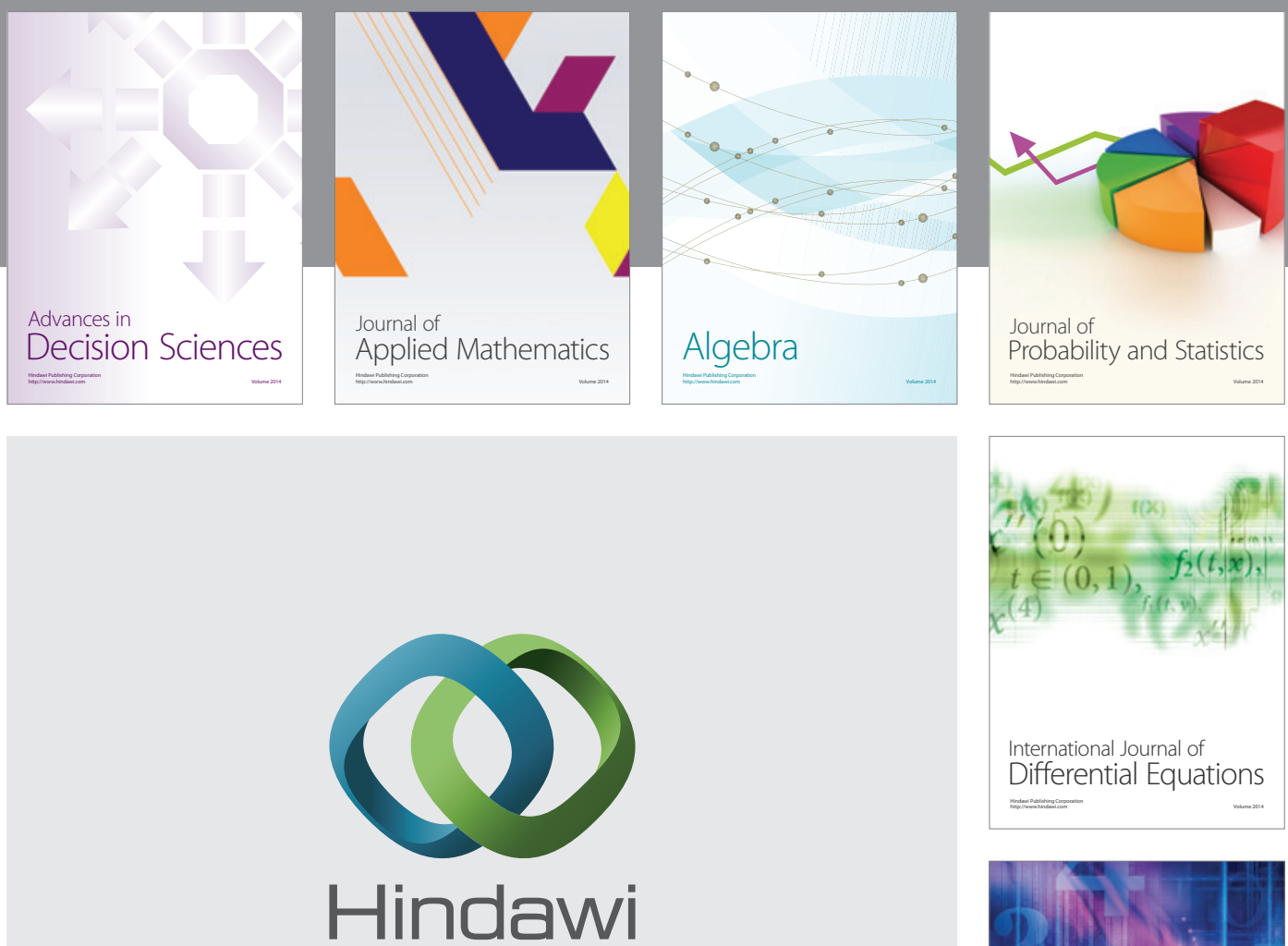

Submit your manuscripts at http://www.hindawi.com
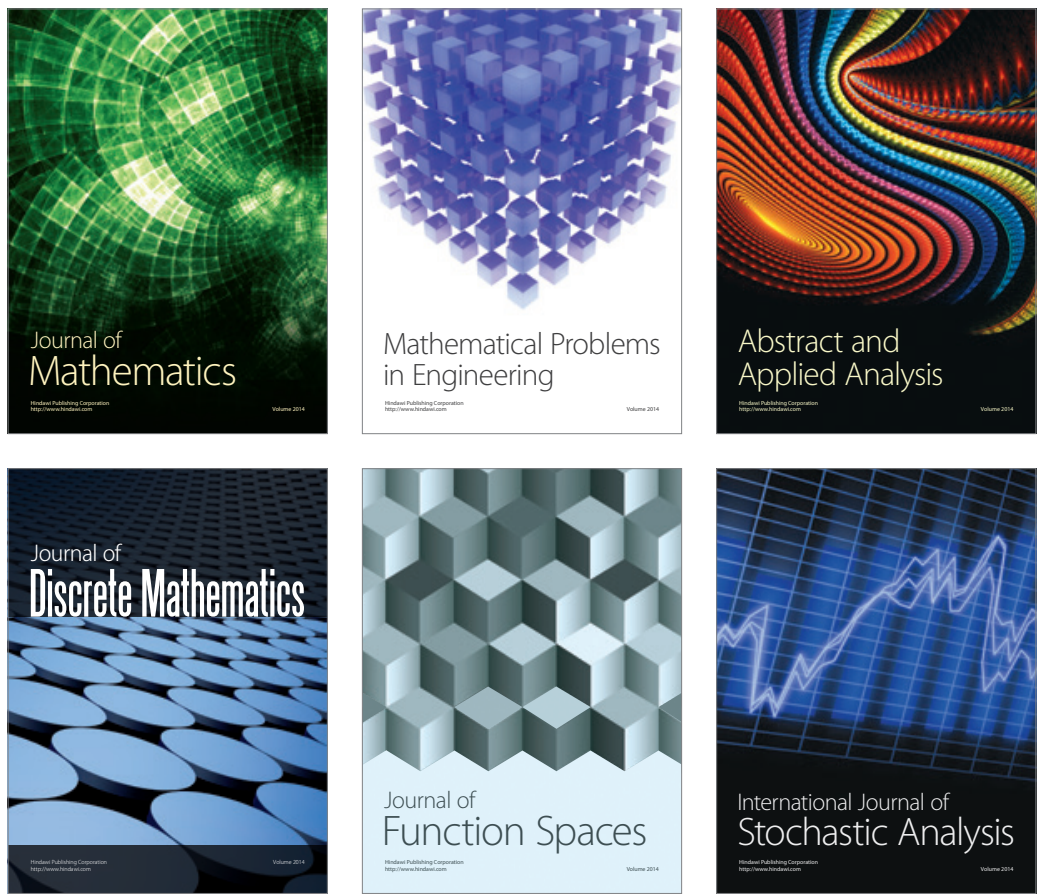

Journal of

Function Spaces

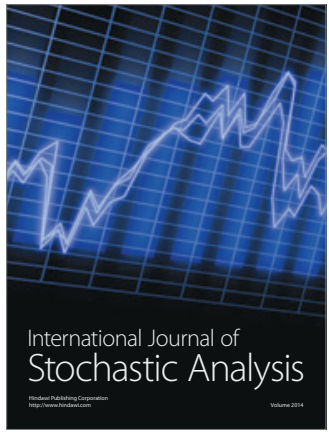

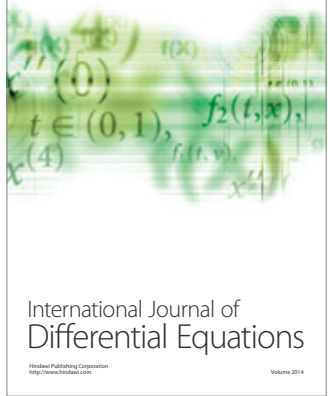
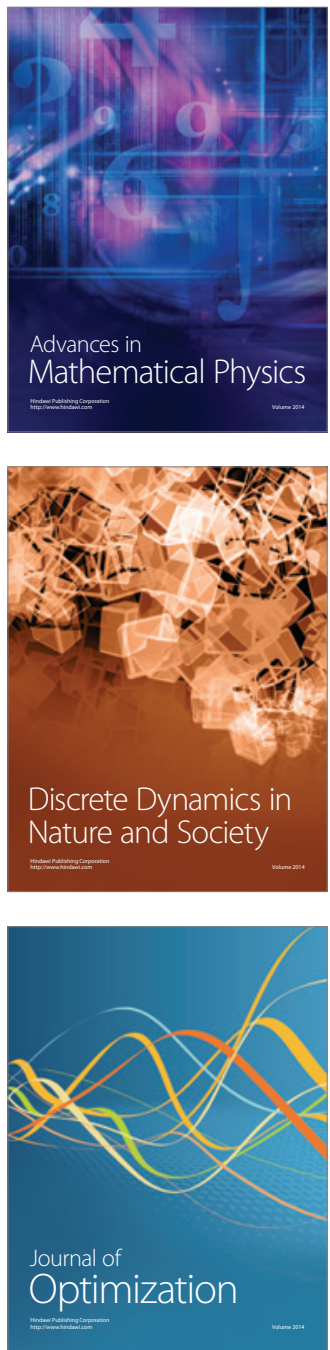